\title{
Immediate allergic reaction to thiocolchicoside confirmed by skin testing and basophil activation test: A case report and literature review
}

\author{
Nucera Eleonora, ${ }^{1, *}$ Inchingolo Riccardo, ${ }^{2, *}$ Ferraironi Manuela, ${ }^{3}$ Aruanno Arianna, ${ }^{1}$ Chini Raffaella, ${ }^{1}$ Rizzi Angela ${ }^{1}$
}

\begin{abstract}
Background: Thiocolchicoside is a muscle relaxant, anti-inflammatory, and analgesic. Administered orally, intramuscularly, or topically, this drug is used in the symptomatic treatment of muscular spasms and rheumatologic disorders. Despite its extensive use, thiocolchicoside is a very rare sensitizer.
\end{abstract}

Objective: To evaluate IgE-mediated reaction to thiocolchicoside by basophil activation test.

Methods: Allergological work-up with skin prick tests, intradermal tests and basophil activation test with thiocolchicoside.

Results: We report the first case of immediate reaction to thiocolchicoside confirmed by basophil activation test in addition to positive skin tests.

Conclusions: BAT can be considered a complementary diagnostic tool to demonstrate an IgE-mediated reaction also for muscle relaxant drugs.

Key words: Thiocolchicoside; drug hypersensitivity; basophil activation test; immediate allergic reaction; CD63+ basophils; central muscle relaxant.

From:

UOSA Allergologia ed Immunologia Clinica, Fondazione Policlinico Universitario A. Gemelli IRCCS, Roma, Italia

2 UOC Pneumologia, Fondazione Policlinico Universitario A. Gemelli IRCCS, Roma, Italia

UOC Microbiologia, Fondazione Policlinico Universitario A. Gemelli IRCCS, Roma, Italia

* Authors equally contributed to this work

Corresponding author:

Angela Rizzi

UOSA Allergologia ed Immunologia Clinica, Fondazione Policlinico

Universitario A. Gemelli IRCCS, Roma, Italia

Largo F. Vito, 1 - 00168 Rome, Italy

E-mail: angela.rizzi@policlinicogemelli.it

\author{
Abbreviations: \\ THC Thiocolchicoside \\ GABA $\gamma$-aminobutyric acid \\ BAT basophil activation test \\ NSAIDs nonsteroidal anti-inflammatory drugs \\ SPTs skin prick tests \\ IDTs intradermal tests \\ fMLP N-Formylmethionyl-leucyl-phenylalanine
}

\section{Background}

Thiocolchicoside (THC) is a semisynthetic sulfur derivative of colchicoside, which has been shown to interact with $\gamma$-aminobutyric acid (GABA) - ergic and glycinergic receptors. ${ }^{1}$

THC is a muscle relaxant agent with anti-inflammatory and analgesic actions. It is also used topically for the treatment of muscular spasms and for rheumatologic, orthopedic and traumatologic disorders. THC is usually well tolerated, but it may occasionally cause adverse effects such as gastrointestinal symptoms, seizures, psychiatric events and, rarely, anaphylaxis..$^{2-4}$

The basophil activation test (BAT) upon the expression of CD63 is an additional tool in the diagnosis of drug allergy that is safer than a provocation test and, in some instances, is the only available diagnostic tool. ${ }^{5}$

\section{Case report}

We report the first case of immediate reaction to THC confirmed by BAT in addition to positive skin tests. 
A 59-year old woman $(158 \mathrm{~cm}, 72 \mathrm{Kg})$ experienced a systemic reaction, characterized by generalized urticaria, labial angioedema and throat constriction, without bronchospasm or shock (grade 2 of Sampson's score of anaphylaxis), 15 minutes after intramuscular administration of diclofenac (Voltaren ${ }^{\oplus}, 75 \mathrm{mg} / 3 \mathrm{ml}$ ) and thiocolchicoside (Miotens ${ }^{\oplus}, 4 \mathrm{mg} / 2 \mathrm{ml}$ ).

She was treated in Emergency Department with intravenous corticosteroids (Metilprednisolone, Urbason ${ }^{\circledR}, 80$ $\mathrm{mg}$ ) and intramuscular antihistamine (Chlorpheniramine, Trimeton ${ }^{\circledast}, 10 \mathrm{mg} / \mathrm{ml}$ ) and she was discharged at home after $24 \mathrm{~h}$ with oral corticosteroid prescription (Metilprednisolone, Medrol $^{\circledR}, 16 \mathrm{mg}$ twice daily for 3 days, then gradual downward titration, halving the dose every 3 days).

Her medical history included ulcerative colitis and rheumatoid arthritis treated with mesalazine, etanercept and methotrexate and occasionally with nonsteroidal anti-inflammatory drugs (NSAIDs). The patient had no known allergies.

She was referred to our Allergy Unit. An allergological work-up, including skin prick tests (SPTs), intradermal tests (IDTs), BAT with diclofenac and THC, was timed for 6 weeks after reaction to minimize the risk of false-negative results after receiving patient's written consent. SPTs were performed with undiluted diclofenac $(25 \mathrm{mg} / \mathrm{ml})$ and thiocolchicoside $(2 \mathrm{mg} / \mathrm{ml}$ ) with saline as the only excipient. After 20 minutes, we observed a positive reaction to THC (mean wheal $20 \mathrm{~mm}$ ) [Figure 1].

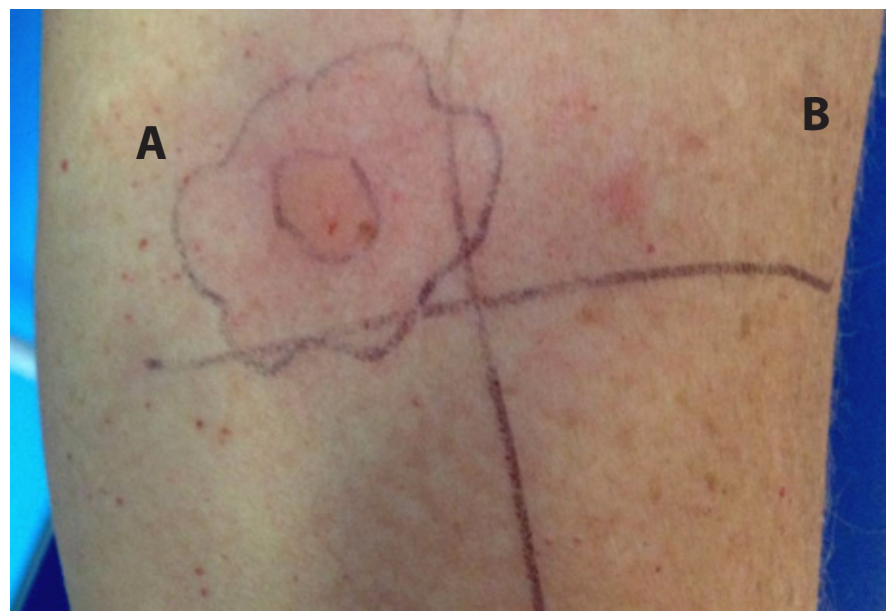

Figure 1 .

IDTs were performed with diluted drugs starting from $0.0002 \mathrm{mg} / \mathrm{ml}$ for THC solution and $0.025 \mathrm{mg} / \mathrm{ml}$ for $\mathrm{di}$ clofenac solution. We recorded a positive area (mean wheal 9 $\mathrm{mm}$ ) for THC 20' after performing the first IDT (0.0002 mg/ $\mathrm{ml})$.

SPT $(25 \mathrm{mg} / \mathrm{ml})$ and IDT $(0.025-25 \mathrm{mg} / \mathrm{ml})$ with diclofenac were negative.

Histamine $(10 \mathrm{mg} / \mathrm{ml})$ and saline were used as positive and negative controls, respectively. IDTs with THC were also performed with negative results in 10 healthy controls, after written informed consent, excluding an irritative reaction to this molecule.
SPTs were performed and read according to the indications of the European Academy of Allergy and Clinical Immunology. ${ }^{5}$

A drug provocation test (DPT) with THC was not performed because of the severity of the index reactions (grade 2 of Sampson's score of anaphylaxis). Furthermore, a DPT with alternative NSAIDs was not performed because, after the anaphylaxis episode, the patient independently took diclofenac by intramuscular route without adverse reactions and reported tolerating meloxicam, orally administered, for arthralgias due to her rheumatoid arthritis.

Taking into account the positive results of skin tests, BAT ${ }^{6}$ was performed with commercially available Flow CAST thiocolchicoside kit according to the manufacturer's instructions (Buhlmann Laboratories AG, Schonenbuch, Switzerland). The Flow CAST determines the basophil activation by measuring the relative amount of CD63+ basophils within the total population of CCR3+ basophils in whole blood. The reagent contains a mixture of monoclonal antibodies to human CD63 labeled with fluorescein isothiocyanate (anti-CD63-FITC) and to human chemokine receptor CCR3 labeled with phycoerythrin (anti-CCR3-PE). As positive control, highly specific monoclonal antibody binding to the unspecific cell activator N-Formylmethionyl-leucyl-phenylalanine (fMLP) was used. The specimen of whole blood of our patient was tested for BAT with THC at different concentrations (dilutions $1 / 10$, $1 / 100,1 / 1000)$ starting from the pure solution for infusion (2 $\mathrm{mg} / \mathrm{ml}$ ). After preliminary dose-finding experiments, the drug concentrations, able to optimally stimulate basophils without cytotoxic effects, ${ }^{7}$ were $0.4,0.04$ and $0.004 \mathrm{mg} / \mathrm{ml}$. Results of BAT are expressed as the percentage of CD63+ basophils. Results of the test are considered as positive when the difference within activated basophils of the patient with and without allergen (negative control) is $\geq 5 \%$. Results of BATs with THC are shown in Figure 2. Basophil activation in the patient was observed at two concentrations $(12 \%$ at $0.04 \mathrm{mg} / \mathrm{ml}$ and $6 \%$ at $0.004 \mathrm{mg} / \mathrm{ml}$ ), while all controls were negative [Figure 2].

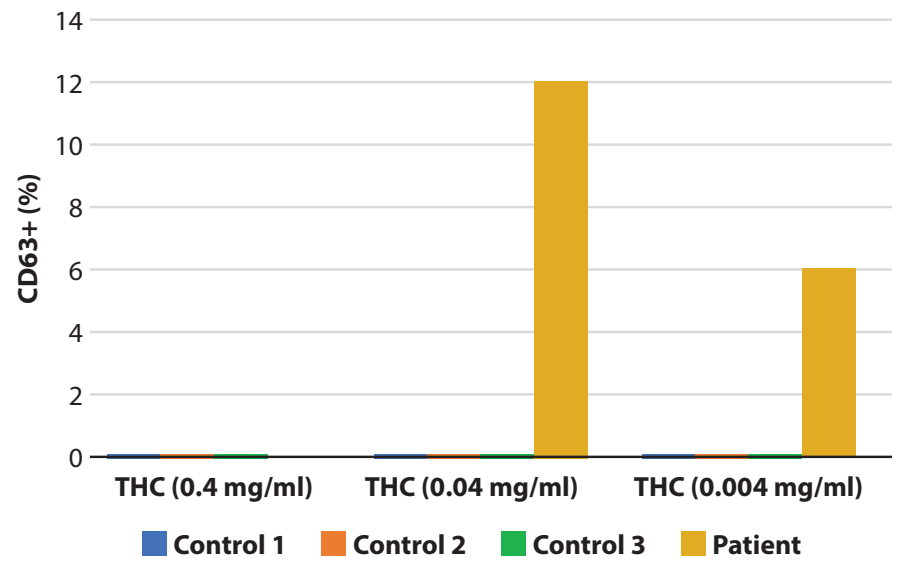

Figure 2. 


\section{Discussion}

THC is a natural glycoside with centrally acting skeletal muscle relaxant effects. ${ }^{8}$ It is an antagonist of GABA receptor function in the central nervous system. ${ }^{8}$ Moreover, it has an agonistic action at the spinal-strychnine-sensitive receptors that can mediate its myorelaxant effect. ${ }^{8}$ Because it is less sedating than other centrally acting muscle relaxants and has anti-inflammatory and analgesic actions, THC is commonly used in the treatment of acute muscle spasms and contractures and also in chronic osteoarticular, rheumatic, and neurologic disorders. ${ }^{8,9}$

THC belongs to the subgroup of spasmolytics, one of the two main groups of drugs referred as skeletal muscle relaxants. ${ }^{10}$ Skeletal muscle relaxants are structurally distinct drugs prescribed for reducing muscle spasms, pain, and hyperreflexia. They are classified into two main groups: neuromuscular blockers and spasmolytics. ${ }^{10}$ Spasmolytics are usually referred as centrally acting muscle relaxants given by oral route, are of two types namely, anti spasmodic and anti spasticity agents. Site of action of antispasticity agents are on the spinal cord to reduce the muscle tone due to upper motor neuron lesions in the spastic neurological conditions. Baclofen, eperisone, tolperisone and THC are the commonly used anti spastic agents. Furthermore, drugs like diazepam and tizanidine are approved as anti spasmodic - anti spastic agents. ${ }^{11}$

In literature, documented hypersensitivity reactions to THC are uncommon, despite of the extensive use of the drug. ${ }^{1}$ The few published cases include: Embolia cutis medicamentosa (or Nicolau syndrome), ${ }^{12}$ immediate and delayed contact dermatitis, ${ }^{13,14}$ photosensitivity reactions ${ }^{15}$ and four cases of immediate anaphylaxis after administration of THC. ${ }^{3}$ In these reports, an IgE-mediated reaction to THC is demonstrated exclusively by positive skin test results (SPTs and IDTs).

To our knowledge, we reported the first case of immediate reaction to THC confirmed by BAT in addition to positive skin tests.

In order to explore if BAT could be a functional in vitro test for the diagnosis of immediate-type drug hypersensitivity also to other centrally acting muscle relaxants, a review of literature was carried out. Pubmed and Web of science were searched up to March 2020.

Primary screening was performed using the following MeSH headings and keywords: "skeletal muscle relaxants", "muscle relaxants, central", "centrally acting muscle relaxants", "baclofen", "eperisone", "tolperisone", "inaperisone", "lanperisone", "silperisone", "diazepam" and "tizanidine". Secondary screening was performed using terms such as "drug hypersensitivity", "drug hypersensitivity, immediate" and "anaphylaxis". Tertiary screening for identifying cases of IgE mediated hypersensitivity to before mentioned drugs. Only English language reports were included. From each report, we retrieved data regarding culprit drug, age, sex, clinical manifestations and allergological diagnostic tests (SPTs, IDTs, drug provocation test and BAT). The selected relevant papers are summarized in Table 1.

No study reports IgE-mediated reactions to baclofen, a GABA derivative that is a specific agonist of GABA-B receptors, used in the treatment of muscle spasticity, especially that due to spinal cord injuries.
More data are available about the IgE-mediated reactions to propiophenones with properties of central muscle relaxants (eperisone, inaperisone, lanperisone, silperisone and tolperisone). Recently, Shin B. and co-workers investigated the clinical manifestations of eperisone-induced immediate-type hypersensitivity and evaluated the role of IDT. The Researchers retrospectively reviewed medical records from 23 patients diagnosed as eperisone-induced immediate-type hypersensitivity with certain or probable causality. Immediate-type hypersensitivity reactions to eperisone, confirmed by IDT and/ or DPT, occurred in 17 patients. ${ }^{16}$ In the same year, Park K.H. et al. reviewed eperisone-related pharmacovigilance data reported in Korea from 2010 to 2015. Moreover, 7-year data reported in a single center were also reviewed. In the single center study, there were 11 patients with eperisone-induced anaphylaxis. All the patients underwent DPT and all the provoked patients showed a positive reaction. Four of the 11 patients with anaphylaxis also underwent SPT and BAT, which were all negative. ${ }^{17}$ Previously, Miki Y. et al. reported a case of eperisone-induced anaphylaxis, in which SPT and BAT were negative. An oral challenge test was necessary to diagnose eperisone-induced acute hypersensitivity. ${ }^{18}$ Even before, in 2012, Hur G.Y. and colleagues described three allergic reactions caused by eperisone and afloqualone. An open-label oral challenge test was performed with each drug to confirm which drugs caused the systemic reactions. To confirm the diagnosis, SPTs, IDT and BAT were performed. ${ }^{19}$ No study reports IgE-mediated reactions to inaperisone, lanperisone and silperisone. Finally, there are few reports of hypersensitivity due to tolperisone. The first case was described by Aleksandrov in 1974. In 2003, Ribi C. et al. described four patients with anaphylaxis attributed to the intake of tolperisone hydrochloride. ${ }^{20}$ In the same year, Kwasniewski et al. described the first case in Poland of anaphylactic shock caused by tolperisone. More recently, Glück J. et al. reported another case of anaphylaxis due to tolperisone confirmed by DPT. ${ }^{21}$

Diazepam, a member of benzodiazepines used since the 1960s for sedation, anterograde amnesia, anxiolysis, as well as treatment of seizures and drug-associated agitation, is a spasmolytic drug with action on central nervous system. In 1977, what was considered to be the first true anaphylaxis to diazepam was published in the British Medical Journal. ${ }^{22}$ The mechanism was attributed to a common metabolite, desmethyldiazepam, the antigenic moiety for cross-allergenicity in benzodiazepines. ${ }^{22} \mathrm{~A}$ recent review on published reports involving anaphylactic or anaphylactoid reactions to benzodiazepines highlights the diagnostic challenge of allergy to benzodiazepines. ${ }^{23}$ In fact, although challenge testing remains the gold standard for diagnosis, it is not routinely performed. In 2002, Asero R. reported a case of diazepam allergy: a healthy 28-year-old nurse with no significant past medical history or allergies presenting for gastroscopy. The patient showed signs of generalized urticaria and shock requiring treatment. ${ }^{24}$

Tizanidine, a congener of clonidine, is a myotonolytic agent FDA-approved for the management of spasticity. ${ }^{25} \mathrm{We}$ did not find in the literature any cases of hypersensitivity to tizanidine. 


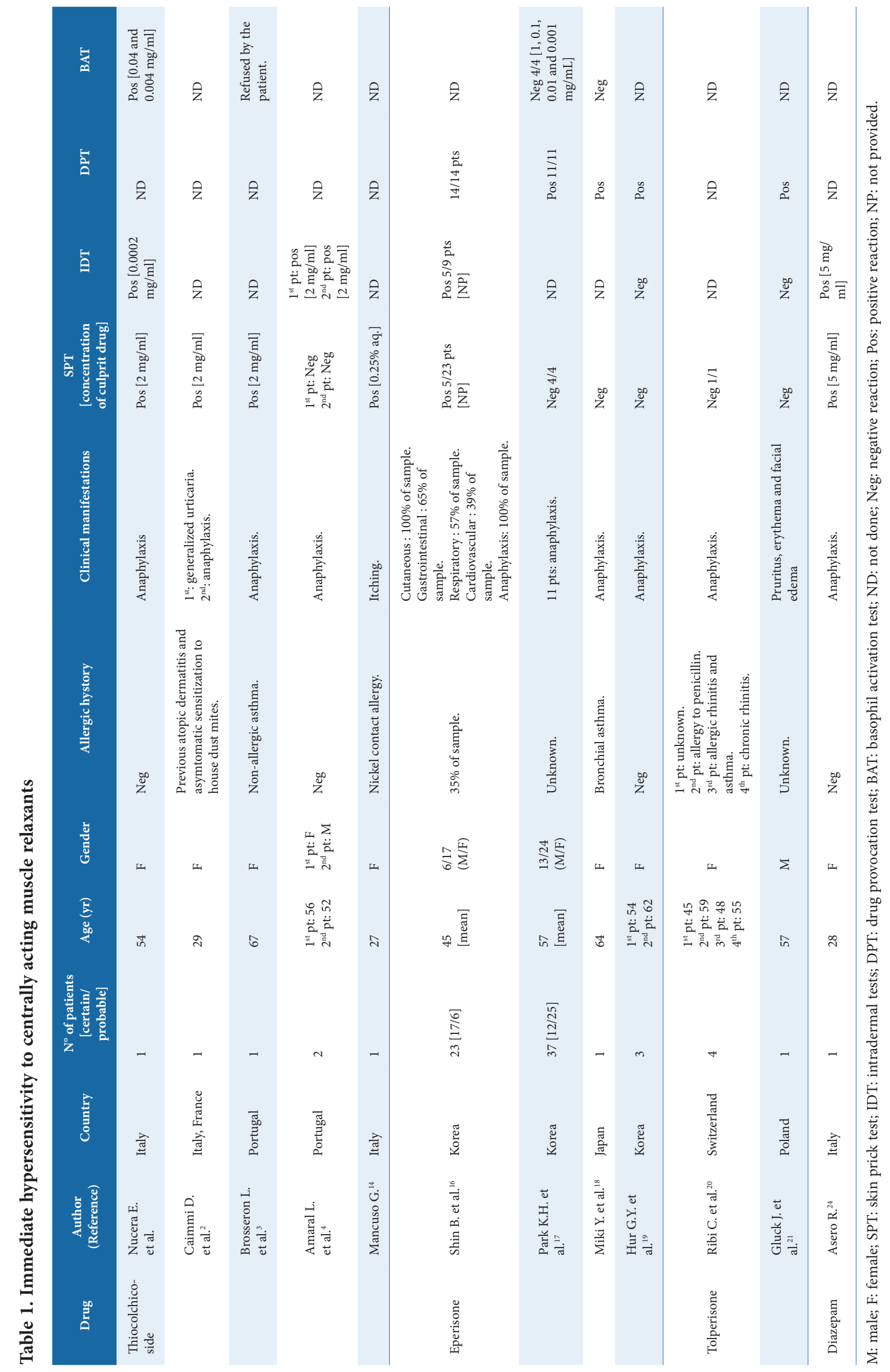


The not negligible number of reported cases of immediate-type drug hypersensitivity to centrally acting muscle relaxants, properly diagnosed and detailed in Table 1, highlights: 1) the high prevalence of the phenomenon in the female population, 2) the severity of reaction to culprit drug (anaphylaxis is frequently reported), and, 3) the heterogeneity of allergological work-up, partly influenced by clinical history and severity of the reaction (BAT was reported only in three reports).

According to a recent review, ${ }^{6}$ BAT have promising results in immediate hypersensitivity reactions to several classes of drugs such as pyrazolones, neuromuscular blockers, beta-lactams and platinum compounds, all examples of classical IgE-mediated hypersensitivity drug reactions, while its usefulness is lower for NSAIDs and quinolones, that degranulate mast cells through non-IgE-mediated mechanism.

Our case demonstrates that BAT can be considered a valid diagnostic tool to investigate an IgE-mediated reaction also for muscle relaxant drugs especially for patients who experienced severe reactions and when the diagnosis cannot be established by serum-specific IgE.

In summary, the combination of multiple methods, such as skin tests and BAT, could improve the diagnostic accuracy for THC-induced anaphylaxis. Although further studies should be performed to accumulate evidence showing the effectiveness of BAT, our case represents the first experience focused on the possible role of BAT to get the diagnosis of hypersensitivity reactions to muscle relaxant drugs, with the advantage of safety for patient avoiding provocation test.

\section{Funding}

The authors declare that no funding was received for the present study.

\section{Conflicts of Interest}

The authors declare that they have no conflicts of interest.

\section{References}

1. Janbroers JM. Review of the toxicology, pharmacodynamics and pharmacokinetics of thiocolchicoside, a GABA-agonist muscle-relaxant with anti-inflammatory and analgesic actions. Acta Ther. 1987;13:221-50.

2. Caimmi D, Caviglioli S, Raschetti R, Demoly P. An IgE immediate reaction to thiocolchicoside. Int J Immunopathol Pharmacol. 2012;25(1):267-8.

3. Brosseron L, Malheiro D, Cadinha S, Moreira da Silva JP. Thiocolchicoside induced anaphylaxis. Allergy. 2015;70(Suppl. 101):331-2.

4. Amaral L, Carolino F, Carneiro-Leão L, Dias de Castro E, Cernadas JR. Thiocolchicoside: An unusual suspect of drug hypersensitivity. J Allergy Clin Immunol Pract. 2017;5(6):1750-1.
5. Brockow K, Garvey LH, Aberer W, Atanaskovic-Markovic M, Barbaud A, Bilo MB, et al. ENDA/EAACI Drug Allergy Interest Group. Skin test concentrations for systemically administered drugs -- an ENDA/EAACI Drug Allergy Interest Group position paper. Allergy. 2013;68:702-12.

6. Campos L, Galvão VR, Kalil J, Castells M, Giavina-Bianchi P. BAT in the Diagnosis of Drug Allergy: a Novel Tool in Clinical Daily Practice? Curr Allergy Asthma Rep. 2019;19(4):20.

7. Mayorga C, Ebo DG, Lang DM, Pichler WJ, Sabato V, Park MA, et al. Controversies in drug allergy: In vitro testing. J Allergy Clin Immunol. 2019;143(1):56-65.

8. Umarkar A, Bavaskar S, Yewale P. Thiocolchicoside as muscle relaxant: a review. History. 2011;8:11-2.

9. Kamath A. Thiocolchicoside: a review. DHR Int J Med Sci. 2013;4:39-45.

10. van Tulder MW, Touray T, Furlan AD, Solway S, Bouter LM; Cochrane Back Review Group. Muscle relaxants for nonspecific low back pain: A systematic review within the framework of the Cochrane Collaboration. Spine. 2003;28:1978-92.

11. Witenko C, Moorman-Li R, Motycka C, Duane K, Hincapie-Castillo J, Leonard $\mathrm{P}$, et al. Considerations for the appropriate use of skeletal muscle relaxants for the management of acute low back pain. Pharma Therapeutics. 2014;39(6):427.

12. Guarneri C, Polimeni G, Guarneri F, Cuzzocrea S. Embolia cutis medicamentosa following thiocolchicoside injection. J Eur Acad Dermatol Venereol. 2008;22:1005-6.

13. Foti C, Cassano N, Mazzarella F, Bonamonte D, Veña GA. Contact allergy to thiocolchicoside. Contact Dermatitis. 1997;37:134.

14. Mancuso G. Immediate and delayed contact hypersensitivity to thiocolchicoside. Acta Dermatovenerologica. 2018;27:203-5.

15. Foti C, Vena GA, Angelini G. Photocontact allergy due to thiocolchicoside. Contact Dermatitis. 1992;27:201-2.

16. Shin B, Yoon SY, Lee JH, Won HK, An J, Kang Y, et al. Clinical characteristics of eperisone-induced immediate-type hypersensitivity. Asian Pac J Allergy Immunol. 2019;10.12932/AP-211018-0420.

17. Park KH, Lee SC, Yuk JE, Kim SR, Lee JH, Park JW. Eperisone-Induced Anaphylaxis: Pharmacovigilance Data and Results of Allergy Testing. Allergy Asthma Immunol Res. 2019;11(2):231-40.

18. Miki Y, Washio K, Masaki T, Nakata K, Fukunaga A, Nishigori C. A case of eperisone hydrochloride-induced anaphylaxis: A true type I reaction? Allergol Int. 2017;66(1):152-3.

19. Hur GY, Hwang EK, Moon JY, Ye YM, Shim JJ, Park HS, et al. Oral muscle relaxant may induce immediate allergic reactions. Yonsei Med J. 2012;53(4):863-5.

20. Ribi C, Vermeulen C, Hauser C. Anaphylactic reactions to tolperisone (Mydocalm). Swiss Med Wkly. 2003;133(25-26):369-71.

21. Glück J, Rymarczyk B, Rogala B. An immediate hypersensitivity reaction caused by tolperisone hydrochloride. J Investig Allergol Clin Immunol. 2011;21(5):411-2.

22. Majumdar SK. Allergy to diazepam. Br Med J. 1977;1:444.

23. Haybarger E, Young AS, Giovannitti JA Jr. Benzodiazepine Allergy With Anesthesia Administration: A Review of Current Literature. Anesth Prog. 2016;63(3):160-7.

24. Asero R. Hypersensitivity to diazepam. Allergy. 2002;57(12):1209.

25. Wagstaff AJ, Bryson HM. Tizanidine. A review of its pharmacology, clinical efficacy and tolerability in the management of spasticity associated with cerebral and spinal disorders. Drugs. 1997;53(3):435-52. 